\title{
Approximate Controllability of Fractional Neutral Evolution Equations in Banach Spaces
}

\author{
N. I. Mahmudov \\ Eastern Mediterranean University, Gazimagusa, TRNC, 10 Mersin, Turkey \\ Correspondence should be addressed to N. I. Mahmudov; nazim.mahmudov@emu.edu.tr
}

Received 24 December 2012; Revised 13 March 2013; Accepted 13 March 2013

Academic Editor: Juan J. Nieto

Copyright (c) 2013 N. I. Mahmudov. This is an open access article distributed under the Creative Commons Attribution License, which permits unrestricted use, distribution, and reproduction in any medium, provided the original work is properly cited.

\begin{abstract}
We discuss the approximate controllability of semilinear fractional neutral differential systems with infinite delay under the assumptions that the corresponding linear system is approximately controllable. Using Krasnoselkii's fixed-point theorem, fractional calculus, and methods of controllability theory, a new set of sufficient conditions for approximate controllability of fractional neutral differential equations with infinite delay are formulated and proved. The results of the paper are generalization and continuation of the recent results on this issue.
\end{abstract}

\section{Introduction and Preliminaries}

Many social, physical, biological and engineering problems can be described by fractional partial differential equations. In fact, fractional differential equations are considered as an alternative model to nonlinear differential equations. In the last two decades, fractional differential equations (see, e.g., Samko et al. [1] and references therein) have attracted many scientists, and notable contributions have been made to both theory and applications of fractional differential equations.

Nowadays, controllability theory for linear systems has already been well established, for finite and infinite dimensional systems; see, for instance, [2]. Several authors have extended these concepts to infinite-dimensional systems represented by nonlinear evolution equations in infinitedimensional spaces, see [3-30]. On the other hand, approximate controllability problems for fractional evolution equations in Hilbert spaces are not yet sufficiently investigated, and there are only few works on it [13-21, 28-30]. So far, the overwhelming majority of the approximate controllability results have only been available for semilinear evolution differential systems in Hilbert spaces, with the exception of the case of [11]. Motivated by the fact that many partial fractional differential equations can be converted into fractional PDE in some Banach spaces, we consider that there is a realistic need to discuss the approximate controllability problem of fractional-order differential systems in Banach spaces. Note that our results are new even for the approximate controllability of fractional neutral differential equations with infinite delay in Hilbert spaces.

Consider the following fractional neutral evolution differential system with infinite delay:

$$
\begin{aligned}
\frac{d^{\alpha}}{d t^{\alpha}}\left[x(t)-h\left(t, x_{t}\right)\right] & =-A x(t)+B u(t)+f\left(t, x_{t}\right), \\
t \in[0, T], & \\
x(t) & =\phi(t) \in B_{l},
\end{aligned}
$$

where the state $x$ takes values in a Banach space $X$ and the control function takes values in a Hilbert space $U$. The functions $h, f$ will be specified in the sequel. Let $x_{t}(\cdot)$ denote $x_{t}(\theta)=x(t+\theta), \theta \in(-\infty, 0]$. Assume that $l:(-\infty, 0] \rightarrow$ $(-\infty, 0)$ is a continuous function satisfying $l=\int_{-\infty}^{0} l(t) d t<$ $\infty$. The Banach space $\left(B_{l},\|\cdot\|_{l}\right)$ induced by function $h$ is defined as follows:

$$
B_{l}:=\left\{\begin{array}{l}
\varphi:(-\infty, 0] \longrightarrow X: \text { for any } c>0 \\
\varphi(\theta) \text { is a bounded and measurable } \\
\text { function on }[-c, 0], \\
\text { and } \int_{-\infty}^{0} l(t) \sup _{t \leq \theta \leq 0}\|\varphi(\theta)\| d t<\infty
\end{array}\right\}
$$


endowed with the norm $\|\varphi\|_{B_{l}}:=\int_{-\infty}^{0} h(t) \sup _{t \leq \theta \leq 0}\|\varphi(\theta)\| d t$. It should be mentioned that (approximate) controllability results for first- and second-order partial neutral functional differential equations with infinite delay were considered by Sakthivel et al. [18], Chalishajar [8], and Chalishajar and Acharya [9].

Throughout this paper, unless otherwise specified, the following notations will be used. Let $(X,\|\cdot\|)$ be a separable reflexive Banach space, and let $\left(X^{*},\|\cdot\|_{*}\right)$ stand for its dual space with respect to the continuous pairing $\langle\cdot, \cdot\rangle$. We may assume, without loss of generality, that $X$ and $X^{*}$ are smooth and strictly convex, by virtue of renorming theorem (see, e.g., $[10])$. In particular, this implies that the duality mapping $J$ of $X$ into $X^{*}$ given by the following relations:

$$
\|J(z)\|_{*}=\|z\|, \quad\langle J(z), z\rangle=\|z\|^{2}, \quad \forall z \in X
$$

is bijective, homogeneous, and demicontinuous, that is, continuous from $X$ with a strong topology into $X^{*}$ with weak topology and strictly monotonic. Moreover, $J^{-1}: X^{*} \rightarrow X$ is also duality mapping.

In this paper, we also assume that $-A: D(A) \subset X \rightarrow X$ is the infinitesimal generator of a compact analytic semigroup $S(t), t>0$, of uniformly bounded linear operator in $X$, that is, there exists $M>1$ such that $\|S(t)\|_{L(X)} \leq M$ for all $t \geq 0$. Without loss of generality, let $0 \in \rho(A)$, where $\rho(A)$ is the resolvent set of $A$. Then, for any $\beta>0$, we can define $A^{-\beta}$ by

$$
A^{-\beta}:=\frac{1}{\Gamma(\beta)} \int_{0}^{\infty} t^{\beta-1} S(t) d t
$$

It follows that each $A^{-\beta}$ is an injective continuous endomorphism of $X$. Hence, we can define $A^{\beta}:=\left(A^{-\beta}\right)^{-1}$, which is a closed bijective linear operator in $X$. It can be shown that each $A^{\beta}$ has dense domain and that $D\left(A^{\gamma}\right) \subset D\left(A^{\beta}\right)$ for $0 \leq \beta \leq \gamma$. Moreover, $A^{\beta+\gamma} x=A^{\beta} A^{\gamma} x=A^{\gamma} A^{\beta} x$ for every $\beta, \gamma \in R$ and $x \in D\left(A^{\mu}\right)$ with $\mu:=\max (\beta, \gamma, \beta+\gamma)$, where $A^{0}=I$ and $I$ is the identity in $X$.

We denote by $X_{\beta}$ the Banach space of $D\left(A^{\beta}\right)$ equipped with norm $\|x\|_{\beta}:=\left\|A^{\beta} x\right\|$ for $x \in D\left(A^{\beta}\right)$, which is equivalent to the graph norm of $A^{\beta}$. Then, we have $X_{\gamma} \hookrightarrow X_{\beta}$, for $0 \leq$ $\beta \leq \gamma$ (with $X_{0}=X$ ), and the embedding is continuous. Moreover, $A^{\beta}$ has the following basic properties.

Lemma 1 (see [31]). A has the following properties:

(i) $S(t): X \rightarrow X_{\beta}$ for each $t>0$ and $\beta \geq 0$.

(ii) $A^{\beta} S(t) x=S(t) A^{\beta} x$ for each $x \in D\left(A^{\beta}\right)$ and $t \geq 0$.

(iii) For every $t>0, A^{\beta} S(t)$ is bounded in $X$, and there exists $M_{\beta}>0$ such that

$$
\left\|A^{\beta} S(t)\right\| \leq M_{\beta} t^{-\beta}
$$

(iv) $A^{-\beta}$ is a bounded linear operator for $0 \leq \beta \leq 1$ in $X$.
From Lemma $1(\mathrm{iv})$, since $A^{-\beta}$ is a bounded linear operator for $0 \leq \beta \leq 1$, there exists a constant $C_{\beta}$ such that $\left\|A^{-\beta}\right\| \leq C_{\beta}$ for $0 \leq \beta \leq 1$.

Let us recall the following known definitions in fractional calculus. For more details, see [1].

Definition 2. The fractional integral of order $\alpha>0$ with the lower limit 0 for a function $f$ is defined as

$$
I^{\alpha} f(t)=\frac{1}{\Gamma(\alpha)} \int_{0}^{t} \frac{f(s)}{(t-s)^{1-\alpha}} d s, \quad t>0, \alpha>0
$$

provided that the right-hand side is pointwise defined on $[0, \infty)$, where $\Gamma$ is the gamma function.

Definition 3. Riemann-Liouville derivative of order $\alpha$ with the lower limit 0 for a function $f:[0, \infty) \rightarrow R$ can be written as

$$
\begin{array}{r}
{ }^{L} D^{\alpha} f(t)=\frac{1}{\Gamma(n-\alpha)} \frac{d^{n}}{d t^{n}} \int_{0}^{t} \frac{f(s)}{(t-s)^{\alpha+1-n}} d s, \\
t>0, n-1<\alpha<n,
\end{array}
$$

Definition 4. The Caputo derivative of order $\alpha$ for a function $f:[0, \infty) \rightarrow R$ can be written as

$$
\begin{array}{r}
{ }^{c} D^{\alpha} f(t)={ }^{L} D^{\alpha}\left(f(t)-\sum_{k=0}^{n-1} \frac{t^{k}}{k !} f^{(k)}(0)\right), \\
t>0, n-1<\alpha<n .
\end{array}
$$

For $x \in X$, we define two families $\left\{\widetilde{S}_{\alpha}(t): t \geq 0\right\}$ and $\left\{\boldsymbol{\mathcal { U }}_{\alpha}(t): t \geq 0\right\}$ of operators by

$$
\begin{aligned}
& \mathfrak{S}_{\alpha}(t)=\int_{0}^{\infty} \Psi_{\alpha}(\theta) S\left(t^{\alpha} \theta\right) d \theta, \\
& \mathfrak{A}_{\alpha}(t)=\alpha \int_{0}^{\infty} \theta \Psi_{\alpha}(\theta) S\left(t^{\alpha} \theta\right) d \theta,
\end{aligned}
$$

where

$$
\Psi_{\alpha}(\theta)=\frac{1}{\pi \alpha} \sum_{n=1}^{\infty}(-1)^{n-1} \frac{\Gamma(n \alpha+1)}{n !} \sin (n \pi \alpha), \quad \theta \in(0, \infty)
$$

is the function of Wright type defined on $(0, \infty)$ which satisfies

$$
\begin{gathered}
\Psi_{\alpha}(\theta) \geq 0, \quad \int_{0}^{\infty} \Psi_{\alpha}(\theta) d \theta=1, \\
\int_{0}^{\infty} \theta^{\zeta} \Psi_{\alpha}(\theta) d \theta=\frac{\Gamma(1+\zeta)}{\Gamma(1+\alpha \zeta)}, \quad \zeta \in(-1, \infty) .
\end{gathered}
$$

The following lemma follows from the results in [32-34]. 
Lemma 5. The operators $\mathfrak{S}_{\alpha}$ and $\mathfrak{\mathfrak { A }}_{\alpha}$ have the following properties:

(i) for any fixed $t \geq 0$ and any $x \in X_{\beta}$, one has the operators $\mathfrak{S}_{\alpha}(t)$ and $\mathfrak{A}_{\alpha}(t)$ which are linear and bounded operators; that is, for any $x \in X_{\beta}$,

$$
\left\|\widetilde{S}_{\alpha}(t) x\right\|_{\beta} \leq M\|x\|_{\beta}, \quad\left\|\mathfrak{\mathcal { U }}_{\alpha}(t) x\right\|_{\beta} \leq \frac{M}{\Gamma(\alpha)}\|x\|_{\beta} ;
$$

(ii) the operators $\mathfrak{S}_{\alpha}(t)$ and $\mathfrak{A}_{\alpha}(t)$ are strongly continuous for all $t \geq 0$;

(iii) $\mathfrak{S}_{\alpha}(t)$ and $\mathfrak{A}_{\alpha}(t)$ are norm continuous in $X$ for $t>0$;

(iv) $\mathfrak{S}_{\alpha}(t)$ and $\mathfrak{\mathfrak { A }}_{\alpha}(t)$ are compact operators in $X$ for $t>0$;

(v) for every $t>0$, the restriction of $\mathfrak{S}_{\alpha}(t)$ to $X_{\beta}$ and the restriction of $\mathfrak{\mathfrak { A }}_{\alpha}(t)$ to $X_{\beta}$ are norm continuous;

(vi) for every $t>0$, the restriction of $\mathfrak{S}_{\alpha}(t)$ to $X_{\beta}$ and the restriction of $\mathfrak{\mathfrak { A }}_{\alpha}(t)$ to $X_{\beta}$ are compact operators in $X_{\beta}$;

(vii) for all $x \in X$ and $t \in[0, T]$,

$$
\left\|A^{\beta} \mathfrak{\mathcal { T }}_{\alpha}(t) x\right\| \leq C_{\beta} t^{-\alpha \beta}\|x\|, \quad C_{\beta}:=\frac{M_{\beta} \alpha \Gamma(2-\beta)}{\Gamma(1+\alpha(1-\beta))} .
$$

In this paper, we adopt the following definition of mild solution of (1).

Definition 6. A solution $x(\cdot ; u) \in C([0, T], X)$ is said to be a mild solution of $(1)$ if for any $u \in L_{2}([0, T], U)$ the integral equation

$$
\begin{aligned}
x(t)= & \mathfrak{S}_{\alpha}(t)(\phi(0)+g(0, \phi))-g\left(t, x_{t}\right) \\
& -\int_{0}^{t}(t-s)^{\alpha-1} A \mathfrak{A}_{\alpha}(t-s) g\left(s, x_{s}\right) d s \\
& +\int_{0}^{t}(t-s)^{\alpha-1} \mathfrak{A}_{\alpha}(t-s)\left[B u(s)+f\left(s, x_{s}\right)\right] d s
\end{aligned}
$$

is satisfied.

Let $x(T ; u)$ be the state value of $(14)$ at terminal time $T$ corresponding to the control $u$. Introduce the set $\mathfrak{R}(T)=$ $\left\{x(T ; u): u \in L_{2}([0, T], U)\right\}$, which is called the reachable set of the system (14) at terminal time $T$, and its closure in $X$ is denoted by $\overline{\Re(T)}$.

Definition 7. The system (1) is said to be approximately controllable on $[0, T]$ if $\overline{\Re(T)}=X$; that is, given an arbitrary $\varepsilon>0$, it is possible to steer from the point $x_{0}$ to within a distance $\varepsilon$ from all points in the state space $X$ at time $T$.

To investigate the approximate controllability of the system (14), we assume the following conditions.

$\left(\mathrm{H}_{1}\right) A$ is the infinitesimal generator of an analytic semigroup of bounded linear operators $S(t)$ in $X, 0 \in \rho(A)$, $S(t)$ is compact for $t>0$, and there exists a positive constant $M$ such that $\|S(t)\| \leq M$.
$\left(\mathrm{H}_{2}\right)$ The function $g:[0, T] \times B_{l} \rightarrow X$ is continuous, and there exists some constant $M_{g}>0,0<\beta<1$, such that $g$ is $X_{\beta}$-valued and

$$
\begin{gathered}
\left\|A^{\beta} g(t, x)-A^{\beta} g(t, y)\right\| \leq M_{g}\|x-y\|_{B_{l}}, \\
x, y \in B_{l}, t \in[0, T], \\
\left\|A^{\beta} g(t, x)\right\| \leq M_{g}\left(1+\|x\|_{B_{l}}\right) .
\end{gathered}
$$

$\left(\mathrm{H}_{3}\right)$ The function $f:[0, T] \times B_{l} \rightarrow X$ satisfies the following:

(a) $f(t, \cdot): B_{l} \rightarrow X$ is continuous for each $t \in$ $[0, T]$ and for each $x \in B_{l}, f(\cdot, x):[0, T] \rightarrow X$ is strongly measurable;

(b) there is a positive integrable function $n \in$ $L^{\infty}([0, T],[0,+\infty))$ and a continuous nondecreasing function $\Lambda_{f}:[0, \infty) \rightarrow(0, \infty)$ such that for every $(t, x) \in[0, T] \times B_{l}$, we have

$\|f(t, x)\| \leq n(t) \Lambda_{f}\left(\|x\|_{B_{l}}\right), \quad \lim \inf _{r \rightarrow \infty} \frac{\Lambda_{f}(r)}{r}=\sigma_{f}<\infty$.

$\left(\mathrm{H}_{4}\right)$ The following relationship holds:

$$
\begin{aligned}
& \left(1+\frac{1}{\varepsilon} M_{B}^{2} M_{\mathfrak{A}}^{2} \frac{T^{2 \alpha-1}}{2 \alpha-1}\right) \\
& \quad \times\left(M_{g}\left\|A^{-\beta}\right\| l+C_{1-\beta} M_{g} \frac{T^{\alpha \beta}}{\alpha \beta} l+\frac{M}{\Gamma(\alpha)} \frac{T^{\alpha}}{\alpha} \sigma_{f} \sup _{s \in J} n(s)\right)<1 .
\end{aligned}
$$

Here, $M_{B}:=\|B\|, M_{\mathfrak{A}}:=\left\|\mathfrak{A}_{\alpha}\right\|$, and $C_{1-\beta}=\alpha M_{1-\beta} \Gamma$ $(1+\beta) / \Gamma(1+\alpha \beta)$.

$\left(\mathrm{H}_{\mathrm{ac}}\right)$ For every $h \in X z_{\alpha}(h)=\varepsilon\left(\varepsilon I+\Gamma_{0}^{T} J\right)^{-1}(h)$ converges to zero as $\varepsilon \rightarrow 0^{+}$in strong topology, where

$$
\Gamma_{0}^{T}:=\int_{0}^{T}(T-s)^{2(\alpha-1)} \mathfrak{U}_{\alpha}(T-s) B B^{*} \mathfrak{P}_{\alpha}^{*}(T-s) d s
$$

and $z_{\varepsilon}(h)$ is a solution of the equation

$$
\varepsilon z_{\varepsilon}+\Gamma_{0}^{T} J\left(z_{\varepsilon}\right)=\varepsilon h .
$$

Let

$$
C_{T}=\left\{x: x \in C((-\infty, T], X), x_{0}=\phi \in B_{l}\right\} .
$$

set $\|\cdot\|_{T}$ be a seminorm defined by

$$
\|x\|_{T}=\left\|x_{0}\right\|_{B_{l}}+\sup _{0 \leq s \leq T}\|x(s)\|, \quad x \in C_{T} .
$$

Lemma 8 (see [8]). Assume that $x \in C_{T}$, then for all $t \in$ $[0, T], x_{t} \in B_{l}$ and

$$
l\|x(t)\| \leq\left\|x_{t}\right\|_{B_{l}} \leq l \sup _{0 \leq s \leq t}\|x(s)\|+\left\|x_{0}\right\|_{B_{l}} .
$$




\section{Existence Theorem}

In order to formulate the controllability problem in the form suitable for application of fixed-point theorem, it is assumed that the corresponding linear system is approximately controllable. Then, it will be shown that the system (1) is approximately controllable if for all $\varepsilon>0$ there exists a continuous function $x \in C([0, T], X)$ such that

$$
\begin{aligned}
u_{\varepsilon}(t, x) & =(T-t)^{\alpha-1} B^{*} \mathfrak{A}_{\alpha}^{*}(T-t) J\left(\left(\varepsilon I+\Gamma_{0}^{T} J\right)^{-1} p(x)\right), \\
x(t)= & \mathfrak{S}_{\alpha}(t)(\phi(0)+g(0, \phi))-g\left(t, x_{t}\right) \\
& -\int_{0}^{t}(t-s)^{\alpha-1} A \mathfrak{A}_{\alpha}(t-s) g\left(s, x_{s}\right) d s \\
& +\int_{0}^{t}(t-s)^{\alpha-1} \mathfrak{A}_{\alpha}(t-s)\left[B u_{\varepsilon}(s, x)+f\left(s, x_{s}\right)\right] d s
\end{aligned}
$$

where

$$
\begin{aligned}
p(x)= & h-\mathfrak{S}_{\alpha}(T)(\phi(0)+g(0, \phi))+g\left(T, x_{T}\right) \\
& +\int_{0}^{T}(T-s)^{\alpha-1} A \mathfrak{A}_{\alpha}(T-s) g\left(s, x_{s}\right) d s \\
& -\int_{0}^{T}(T-s)^{\alpha-1} \mathfrak{A}_{\alpha}(T-s) f\left(s, x_{s}\right) d s, \quad h \in X .
\end{aligned}
$$

Having noticed this fact, our goal in this section is to find conditions for solvability of (23). Note that it will be shown that the control in (23) drives the system (1) from $\phi(0)$ to

$$
h-\varepsilon J\left(\left(\varepsilon I+\Gamma_{0}^{T} J\right)^{-1} p(x)\right)
$$

provided that the system (23) has a solution.

Theorem 9. Assume that assumptions $\left(H_{1}\right)-\left(H_{4}\right)$ hold and $1 / 2<\alpha<1$. Then, there exists a solution to (23).

Proof. The proof of Theorem 9 follows from Lemmas 10-14 and infinite dimensional analogue of Arzela-Ascoli theorem.

For all $\varepsilon>0$, consider the operator $\Phi_{\varepsilon}: C_{T} \rightarrow C_{T}$ defined as follows:

$$
\begin{aligned}
& \left(\Phi_{\varepsilon} x\right)(t)
\end{aligned}
$$

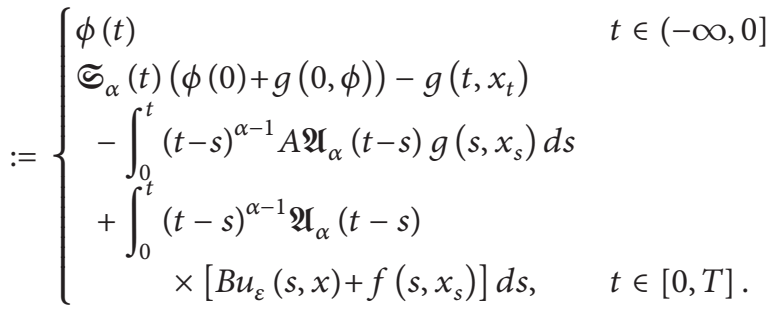

It will be shown that for all $\varepsilon>0$, the operator $\Phi_{\varepsilon}: C_{T} \rightarrow C_{T}$ has a fixed point.

Suppose that $x(t)=\widetilde{\phi}(t)+z(t), t \in(-\infty, T]$, where $\widetilde{\phi}(t)$ is taken as $\phi(t)$ for $t \in(-\infty, 0]$, while for $t \in[0, T]$, it is defined as $\mathfrak{\subseteq}_{\alpha}(t) \phi(0)$. Set

$$
C_{T}^{0}=\left\{z \in C_{T}: z_{0}=0 \in B_{l}\right\}
$$

For any $z \in C_{T}^{0}$, we have

$$
\|z\|_{T}=\left\|z_{0}\right\|_{B_{l}}+\sup _{0 \leq s \leq T}\|z(s)\|=\sup _{0 \leq s \leq T}\|z(s)\| .
$$

Thus, $\left(C_{T}^{0},\|\cdot\|_{T}\right)$ is a Banach space. For each positive number $r>0$, set

$$
\mathbf{B}_{r}:=\left\{z \in C_{T}^{0}:\|z\|_{T} \leq r\right\}
$$

It is clear that $B_{r}$ is bounded closed convex set in $C_{T}^{0}$. For any $z \in B_{r}$, we see that

$$
\begin{aligned}
\left\|\tilde{\phi}_{t}+z_{t}\right\|_{B_{l}} \leq & \left\|\tilde{\phi}_{t}\right\|_{B_{l}}+\left\|z_{t}\right\|_{B_{l}} \\
\leq & l \sup _{0 \leq s \leq t}\|\tilde{\phi}(s)\|+\left\|\tilde{\phi}_{0}\right\|_{B_{l}} \\
& +l \sup _{0 \leq s \leq t}\|z(s)\|+\left\|z_{0}\right\|_{B_{l}} \\
& \leq l(M\|\phi(0)\|+r)+\|\phi\|_{B_{l}}:=R(r) .
\end{aligned}
$$

Consider the maps $\Pi_{\varepsilon}, \Theta_{\varepsilon}: C_{T}^{0} \rightarrow C_{T}^{0}$ defined by

$\left(\Pi_{\varepsilon} z\right)(t)$

$:= \begin{cases}0 & t \in(-\infty, 0], \\ \mathfrak{S}_{\alpha}(t) g(0, \phi)-g\left(t, \widetilde{\phi}_{t}+z_{t}\right) & \\ -\int_{0}^{t}(t-s)^{\alpha-1} A \mathfrak{A}_{\alpha}(t-s) g\left(s, \widetilde{\phi}_{s}+z_{s}\right) d s & t \in[0, T],\end{cases}$

$\left(\Theta_{\varepsilon} z\right)(t)$

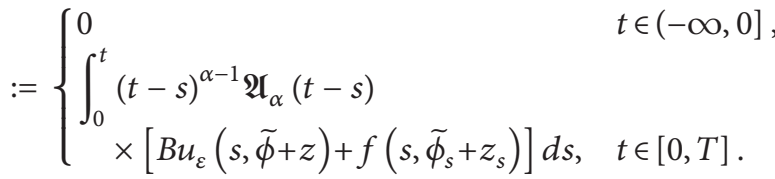

Obviously, the operator $\Phi_{\varepsilon}$ has a fixed point if and only if operator $\Pi_{\varepsilon}+\Theta_{\varepsilon}$ has a fixed point. In order to prove that $\Pi_{\varepsilon}+\Theta_{\varepsilon}$ has a fixed point we will employ the Krasnoselskii's fixed-point theorem.

Lemma 10. Under assumptions $\left(H_{1}\right)-\left(H_{4}\right)$, for any $\varepsilon>0$, there exists a positive number $r:=r(\varepsilon)$ such that $\Pi_{\varepsilon} z_{r}+\Theta_{\varepsilon} y_{r} \subset$ $\mathbf{B}_{r}$ for all $z, y \in \mathbf{B}_{r r}$. 
Proof. Let $\varepsilon>0$ be fixed. If it is not true, then for each $r>0$, there are functions $z_{r}, y_{r} \in B_{r}$, but $\Pi_{\varepsilon}\left(z_{r}\right)+\Theta_{\varepsilon}\left(y_{r}\right) \notin \mathbf{B}_{r}$. So for some $t=t(r) \in[0, T]$, one can show that

$$
\begin{aligned}
r \leq & \left\|\Pi_{\varepsilon}\left(z_{r}\right)(t)+\Theta_{\varepsilon}\left(y_{r}\right)(t)\right\| \\
\leq & \left\|\mathfrak{S}_{\alpha}(t) g(0, \phi)\right\|+\left\|g\left(t, \widetilde{\phi}_{t}+z_{t}\right)\right\| \\
& +\left\|\int_{0}^{t}(t-s)^{\alpha-1} A \mathfrak{A}_{\alpha}(t-s) g\left(s, \widetilde{\phi}_{s}+z_{s}\right) d s\right\| \\
& +\left\|\int_{0}^{t}(t-s)^{\alpha-1} \mathfrak{A}_{\alpha}(t-s) f\left(s, \widetilde{\phi}_{s}+y_{s}\right) d s\right\| \\
& +\left\|\int_{0}^{t}(t-s)^{\alpha-1} \mathfrak{A}_{\alpha}(t-s) B u_{\varepsilon}(s, \phi+y) d s\right\| \\
= & : I_{1}+I_{2}+I_{3}+I_{4}+I_{5} .
\end{aligned}
$$

Let us estimate $I_{i}, i=1, \ldots, 4$. By the assumption $\left(\mathrm{H}_{2}\right)$, we have

$$
\begin{aligned}
I_{1} & \leq M\left\|A^{-\beta}\right\|\left\|A^{\beta} g(0, \phi)\right\| \leq M M_{g}\left\|A^{-\beta}\right\|\left(1+\|\phi\|_{B_{l}}\right), \\
I_{2} & \leq\left\|A^{-\beta}\right\|\left\|A^{\beta} g\left(t, \widetilde{\phi}_{t}+z_{t}\right)\right\| \leq M_{g}\left\|A^{-\beta}\right\|\left(1+\left\|\widetilde{\phi}_{t}+z_{t}\right\|_{B_{l}}\right) \\
& \leq M_{g}\left\|A^{-\beta}\right\|(1+R(r)) .
\end{aligned}
$$

Using Lemma 5 and the Hölder inequality, one can deduce that

$$
\begin{aligned}
I_{3} & \leq \int_{0}^{t}\left\|(t-s)^{\alpha-1} A^{1-\beta} \mathfrak{A}_{\alpha}(t-s) A^{\beta} g\left(s, \widetilde{\phi}_{s}+z_{s}\right)\right\| d s \\
& \leq \frac{M_{1-\beta} \alpha \Gamma(1+\beta)}{\Gamma(1+\alpha \beta)} \int_{0}^{t}(t-s)^{\alpha \beta-1}\left\|A^{\beta} g\left(s, \widetilde{\phi}_{s}+z_{s}\right)\right\| d s \\
& \leq K(\alpha, \beta) \int_{0}^{t}(t-s)^{\alpha \beta-1} M_{g}\left(1+\left\|\widetilde{\phi}_{s}+z_{s}\right\|_{B_{l}}\right) d s \\
& \leq K(\alpha, \beta) M_{g} \frac{T^{\alpha \beta}}{\alpha \beta}(1+R(r)) .
\end{aligned}
$$

Using the assumption $\left(\mathrm{H}_{3}\right)$, one has

$$
\begin{aligned}
I_{4} & \leq \int_{0}^{t}\left\|(t-s)^{\alpha-1} \mathfrak{A}_{\alpha}(t-s) f\left(s, \widetilde{\phi}_{s}+y_{s}\right)\right\| d s \\
& \leq \frac{M}{\Gamma(\alpha)} \int_{0}^{t}(t-s)^{\alpha-1}\left\|f\left(s, \widetilde{\phi}_{s}+y_{s}\right)\right\| d s \\
& \leq \frac{M}{\Gamma(\alpha)} \int_{0}^{t}(t-s)^{\alpha-1} n(s) \Lambda_{f}\left(\left\|\tilde{\phi}_{s}+y_{s}\right\|\right) d s \\
& \leq \frac{M}{\Gamma(\alpha)} \frac{T^{\alpha}}{\alpha} \Lambda_{f}(R(r)) \sup _{s \in J} n(s) .
\end{aligned}
$$

Combining the estimates (32)-(36) yields

$$
\begin{aligned}
I_{1}+I_{2}+I_{3}+I_{4}< & M M_{g}\left\|A^{-\beta}\right\|\left(1+\|\phi\|_{B_{l}}\right) \\
& +M_{g}\left\|A^{-\beta}\right\|(1+R(r)) \\
& +C_{1-\beta} M_{g} \frac{T^{\alpha \beta}}{\alpha \beta}(1+R(r)) \\
& +\frac{M}{\Gamma(\alpha)} \frac{T^{\alpha}}{\alpha} \Lambda_{f}(R(r)) \sup _{s \in J} n(s):=\Delta .
\end{aligned}
$$

On the other hand,

$$
\begin{aligned}
I_{5} \leq & \int_{0}^{t}\left\|(t-s)^{\alpha-1} \mathfrak{A}_{\alpha}(t-s) B u_{\varepsilon}(s, \phi+y)\right\| d s \\
= & \int_{0}^{t} \|(t-s)^{\alpha-1}(T-s)^{\alpha-1} \mathfrak{A}_{\alpha}(t-s) B B^{*} \mathfrak{A}_{\alpha}^{*} \\
& \quad \times(T-t) J\left(\left(\varepsilon I+\Gamma_{0}^{T} J\right)^{-1} p(\phi+y)\right) \| d s \\
\leq & \int_{0}^{t}\left\|(t-s)^{\alpha-1}(T-s)^{\alpha-1} \mathfrak{A}_{\alpha}(t-s) B B^{*} \mathfrak{A}_{\alpha}^{*}(T-t)\right\| d s \\
& \times\left\|J\left(\left(\varepsilon I+\Gamma_{0}^{T} J\right)^{-1} p(\phi+y)\right)\right\| \\
\leq & M_{B}^{2} M_{\mathfrak{A}}^{2} \frac{T^{2 \alpha-1}}{2 \alpha-1}\left\|J\left(\left(\varepsilon I+\Gamma_{0}^{T} J\right)^{-1} p(\phi+y)\right)\right\| \\
= & M_{B}^{2} M_{\mathfrak{Q}}^{2} \frac{T^{2 \alpha-1}}{2 \alpha-1}\left\|\left(\varepsilon I+\Gamma_{0}^{T} J\right)^{-1} p(\phi+y)\right\| \\
\leq & \frac{1}{\varepsilon} M_{B}^{2} M_{\mathfrak{Q}}^{2} \frac{T^{2 \alpha-1}}{2 \alpha-1}\|p(\phi+y)\| \\
\leq & \frac{1}{\varepsilon} M_{B}^{2} M_{\mathfrak{Q}}^{2} \frac{T^{2 \alpha-1}}{2 \alpha-1} \Delta .
\end{aligned}
$$

Thus,

$$
\begin{aligned}
r & \leq\left\|\Pi_{\varepsilon}\left(z_{r}\right)(t)+\Theta_{\varepsilon}\left(y_{r}\right)(t)\right\| \\
& \leq \Delta+\frac{1}{\varepsilon} M_{B}^{2} M_{\mathfrak{Q}}^{2} \frac{T^{2 \alpha-1}}{2 \alpha-1} \Delta \\
& =\left(1+\frac{1}{\varepsilon} M_{B}^{2} M_{\mathfrak{Q}}^{2} \frac{T^{2 \alpha-1}}{2 \alpha-1}\right) \Delta .
\end{aligned}
$$

Dividing both sides of (39) by $r$ and taking $r \rightarrow \infty$, we obtain that

$$
\begin{aligned}
& \left(1+\frac{1}{\varepsilon} M_{B}^{2} M_{\mathfrak{Q}}^{2} \frac{T^{2 \alpha-1}}{2 \alpha-1}\right) \\
& \times\left(M_{g}\left\|A^{-\beta}\right\| l+M_{g} C_{1-\beta} \frac{T^{\alpha \beta}}{\alpha \beta} l+\frac{M}{\Gamma(\alpha)} \frac{T^{\alpha}}{\alpha} \sigma_{f} \sup _{s \in J} n(s)\right) \geq 1,
\end{aligned}
$$

which is a contradiction by assumption $\left(\mathrm{H}_{4}\right)$. Thus, $\Pi_{\varepsilon} z_{r}+$ $\Theta_{\varepsilon} y_{r} \subset \mathbf{B}_{r}$ for some $r>0$. 
Lemma 11. Let assumptions $\left(H_{1}\right)-\left(H_{4}\right)$ hold. Then, $\Theta_{1}$ is contractive.

Proof. Let $x, y \in \mathbf{B}_{r}$. Then,

$$
\begin{aligned}
& \left\|\left(\Pi_{\varepsilon} x\right)(t)-\left(\Pi_{\varepsilon} y\right)(t)\right\| \\
& \leq\left\|g\left(t, \tilde{\phi}_{t}+x_{t}\right)-g\left(t, \tilde{\phi}_{t}+y_{t}\right)\right\| \\
& +\| \int_{0}^{t}(t-s)^{\alpha-1} A \mathfrak{A}_{\alpha}(t-s) \\
& \quad \times\left(g\left(s, \tilde{\phi}_{s}+x_{s}\right)-g\left(s, \tilde{\phi}_{s}+y_{s}\right)\right) d s \| \\
& \leq\left\|A^{-\beta}\right\| M_{g}\left\|x_{t}-y_{t}\right\|_{B_{l}}+C_{1-\beta} \\
& \quad \times \int_{0}^{t}(t-s)^{\alpha \beta-1}\left\|A^{\beta}\left(g\left(s, \tilde{\phi}_{s}+x_{s}\right)-g\left(s, \widetilde{\phi}_{s}+y_{s}\right)\right)\right\| d s \\
& \leq\left\|A^{-\beta}\right\| M_{g}\left\|x_{t}-y_{t}\right\|_{B_{l}}+C_{1-\beta} M_{g} \\
& \quad \times \int_{0}^{t}(t-s)^{\alpha \beta-1}\left\|x_{s}-y_{s}\right\|_{B_{l}} d s .
\end{aligned}
$$

Hence,

$$
\begin{aligned}
& \left\|\left(\Pi_{\varepsilon} x\right)(t)-\left(\Pi_{\varepsilon} y\right)(t)\right\| \\
& \quad \leq M_{g} l\left(\left\|A^{-\beta}\right\|+C_{1-\beta} \frac{T^{\alpha \beta}}{\alpha \beta}\right) \sup _{0 \leq s \leq t}\|x(s)-y(s)\|,
\end{aligned}
$$

where we have used the fact that $x_{0}=y_{0}=0$. Thus,

$$
\begin{aligned}
& \sup _{0 \leq t \leq T}\left\|\left(\Pi_{\varepsilon} x\right)(t)-\left(\Pi_{\varepsilon} y\right)(t)\right\| \\
& \quad \leq M_{g} l\left(\left\|A^{-\beta}\right\|+C_{1-\beta} \frac{T^{\alpha \beta}}{\alpha \beta}\right) \sup _{0 \leq s \leq T}\|x(s)-y(s)\|,
\end{aligned}
$$

so $\Theta_{1}$ is a contraction by assumption $\left(\mathrm{H}_{4}\right)$.

Lemma 12. Let assumptions $\left(H_{1}\right)-\left(H_{4}\right)$ hold. Then, $\Theta_{\varepsilon}$ maps bounded sets to bounded sets in $B_{r}$.

Proof. By the similar argument as Lemma 10, we obtain

$$
\begin{aligned}
\left\|\left(\Theta_{\varepsilon} z\right)(t)\right\|< & \left(1+\frac{1}{\varepsilon} M_{B}^{2} M_{\mathfrak{A}}^{2} \frac{T^{2 \alpha-1}}{2 \alpha-1}\right) \\
& \times \frac{M}{\Gamma(\alpha)} \frac{T^{\alpha}}{\alpha} \Lambda_{f}(R(r)) \sup _{s \in J} n(s):=r_{1}(\varepsilon)
\end{aligned}
$$

which implies that $\Theta_{\varepsilon} z \in \mathbf{B}_{r_{1}(\varepsilon)}$.

Lemma 13. Let assumptions $\left(H_{1}\right)-\left(H_{4}\right)$ hold. Then, the set $\left\{\Theta_{\varepsilon} z: z \in \mathbf{B}_{r}\right\}$ is an equicontinuous family of functions on $[0, T]$.

Proof. Let $0<\eta<t<T$ and $\delta>0$ such that

$$
\left\|\mathfrak{\mathcal { A }}_{\alpha}\left(s_{1}\right)-\mathfrak{\mathfrak { A }}_{\alpha}\left(s_{2}\right)\right\|<\eta
$$

for every $s_{1}, s_{2} \in[0, T]$ with $\left|s_{1}-s_{2}\right|<\delta$. For $z \in \mathbf{B}_{r}, 0<|h|<$ $\delta, t+h \in[0, T]$, we have

$$
\begin{aligned}
& \left\|\left(\Theta_{\varepsilon} z\right)(t+h)-\left(\Theta_{\varepsilon} z\right)(t)\right\| \\
& \leq \| \int_{0}^{t}\left((t+h-s)^{\alpha-1}-(t-s)^{\alpha-1}\right) \mathfrak{\Re}_{\alpha}(t+h-s) \\
& \times\left[(T-s)^{\alpha-1} B v_{\varepsilon}(s, \tilde{\phi}+z)+f\left(s, \tilde{\phi}_{s}+z_{s}\right)\right] d s \| \\
& +\| \int_{t}^{t+h}(t+h-s)^{\alpha-1} \mathfrak{\mathcal { A }}_{\alpha}(t+h-s) \\
& \times\left[(T-s)^{\alpha-1} B v_{\varepsilon}(s, \tilde{\phi}+z)+f\left(s, \tilde{\phi}_{s}+z_{s}\right)\right] d s \| \\
& +\| \int_{0}^{t}(t-s)^{\alpha-1}\left(\mathfrak{A}_{\alpha}(t+h-s)-\mathfrak{A}_{\alpha}(t-s)\right) \\
& \times\left[(T-s)^{\alpha-1} B v_{\varepsilon}(s, \widetilde{\phi}+z)+f\left(s, \widetilde{\phi}_{s}+z_{s}\right)\right] d s \| .
\end{aligned}
$$

Applying (38) and the Hölder inequality, we obtain

$$
\begin{aligned}
& \left\|\left(\Theta_{\varepsilon} z\right)(t+h)-\left(\Theta_{\varepsilon} z\right)(t)\right\| \\
& \leq \frac{M}{\Gamma(\alpha)} \Lambda_{f}(R(r)) \int_{0}^{t}\left((t+h-s)^{\alpha-1}-(t-s)^{\alpha-1}\right) n(s) d s \\
& +\frac{M}{\Gamma(\alpha)} \frac{1}{\varepsilon} M_{B} M_{\mathfrak{A}} \Delta \\
& \quad \times \int_{0}^{t}\left((t+h-s)^{\alpha-1}-(t-s)^{\alpha-1}\right)(T-s)^{\alpha-1} d s \\
& +\frac{M}{\Gamma(\alpha)} \Lambda_{f}(R(r)) \int_{t}^{t+h}(t+h-s)^{\alpha-1} n(s) d s \\
& +\frac{M}{\Gamma(\alpha)} \frac{1}{\varepsilon} M_{B} M_{\mathfrak{A}} \Delta \int_{t}^{t+h}(t+h-s)^{\alpha-1}(T-s)^{\alpha-1} d s \\
& +\frac{\eta T^{\alpha}}{\alpha} \Lambda_{f}(R(r)) \int_{0}^{t}(t-s)^{\alpha-1} n(s) d s \\
& +\frac{\eta T^{\alpha}}{\alpha} \frac{1}{\varepsilon} M_{B} M_{\mathfrak{A}} \Delta \int_{0}^{t}(t-s)^{\alpha-1}(T-s)^{\alpha-1} d s .
\end{aligned}
$$

Therefore, for $\varepsilon$ sufficiently small, the right-hand side of (47) tends to zero as $h \rightarrow 0$. On the other hand, the compactness of $\mathfrak{A}_{\alpha}(t), t>0$ implies the continuity in the uniform operator topology. Thus, the set $\left\{\Theta_{\varepsilon} z: z \in \mathbf{B}_{r}\right\}$ is equicontinuous.

Lemma 14. Let assumptions $\left(H_{1}\right)-\left(H_{4}\right)$ hold. Then, $\Theta_{\varepsilon}$ maps $\mathbf{B}_{r}$ onto a precompact set in $\mathbf{B}_{r}$. 
Proof. Let $0<t \leq T$ be fixed and $\varepsilon$ be a real number satisfying $0<\lambda<t$. For $\delta>0$, define an operator $\Theta_{\varepsilon}^{\lambda, \delta}$ on $B_{r}$ by

$$
\begin{aligned}
& \left(\Theta_{\varepsilon}^{\lambda, \delta} z\right)(t) \\
& =\alpha \int_{0}^{t-\lambda} \int_{\delta}^{\infty} \theta(t-s)^{\alpha-1} \eta_{\alpha}(\theta) S\left((t-s)^{\alpha} \theta\right) \\
& \quad \times\left[B u_{\varepsilon}(s, \tilde{\phi}+z)+f\left(s, \tilde{\phi}_{s}+z_{s}\right)\right] d s \\
& =\alpha S\left(\lambda^{\alpha} \delta\right) \int_{0}^{t-\lambda} \int_{\delta}^{\infty} \theta(t-s)^{\alpha-1} \eta_{\alpha}(\theta) S\left((t-s)^{\alpha} \theta-\lambda^{\alpha} \delta\right) \\
& \quad \times\left[B u_{\varepsilon}(s, \widetilde{\phi}+z)+f\left(s, \widetilde{\phi}_{s}+z_{s}\right)\right] d s .
\end{aligned}
$$

Since $S(t), t>0$ is a compact operator, the set $\left\{\left(\Theta_{\varepsilon}^{\lambda, \delta} z\right)(t): z \in\right.$ $\left.\mathbf{B}_{r}\right\}$ is precompact in $H$ for every $0<\lambda<t, \delta>0$. Moreover, for each $z \in \mathbf{B}_{r}$, we have

$$
\begin{aligned}
& \left\|\left(\Theta_{\varepsilon} z\right)(t)-\left(\Theta_{\varepsilon}^{\lambda, \delta} z\right)(t)\right\| \\
& \leq \alpha E \| \int_{0}^{t} \int_{0}^{\delta} \theta(t-s)^{\alpha-1} \eta_{\alpha}(\theta) S\left((t-s)^{\alpha} \theta\right) \\
& \times\left[B u_{\varepsilon}(s, \tilde{\phi}+z)+f\left(s, \widetilde{\phi}_{s}+z_{s}\right)\right] d \theta d s \| \\
& +\alpha E \| \int_{t-\lambda}^{t} \int_{\delta}^{\infty} \theta(t-s)^{\alpha-1} \eta_{\alpha}(\theta) S\left((t-s)^{\alpha} \theta\right) \\
& \quad \times\left[B u_{\varepsilon}(s, \tilde{\phi}+z)+f\left(s, \tilde{\phi}_{s}+z_{s}\right)\right] d \theta d s \| \\
& :=J_{1}+J_{2} .
\end{aligned}
$$

A similar argument as before

$$
\begin{aligned}
J_{1} \leq & \alpha M \int_{0}^{t}(t-s)^{\alpha-1}\left(\left\|B u_{\varepsilon}(s, \tilde{\phi}+z)\right\|+\left\|f\left(s, \tilde{\phi}_{s}+z_{s}\right)\right\|\right) d s \\
& \times\left(\int_{0}^{\delta} \theta \eta_{\alpha}(\theta) d \theta\right) \\
\leq & \alpha M\left(\frac{1}{\varepsilon} M_{B} M_{\mathfrak{A}} \Delta \int_{0}^{t}(t-s)^{\alpha-1}(T-s)^{\alpha-1} d s\right. \\
J_{2} \leq & \alpha M \int_{t-\lambda}^{t}(t-s)^{\alpha-1}\left(\left\|B u_{\varepsilon}(s, \tilde{\phi}+z)\right\|+\left\|f\left(s, \tilde{\phi}_{s}+z_{s}\right)\right\|\right) d s \\
& \left.\times\left(\int_{\delta}^{\infty} \theta \eta_{\alpha}(\theta) d \theta\right) \int_{0}^{t}(t-s)^{\alpha-1} n(s) d s\right)\left(\int_{0}^{\delta} \theta \eta_{\alpha}(\theta) d \theta\right), \\
\leq & \frac{\alpha M}{\Gamma(1+\alpha)}\left(\frac{1}{\varepsilon} M_{B} M_{\mathfrak{Q}} \Delta \int_{t-\lambda}^{t}(t-s)^{\alpha-1}(T-s)^{\alpha-1} d s\right. \\
& \left.+\Lambda \int_{f}(R(r)) \int_{t-\lambda}^{t}(t-s)^{\alpha-1} n(s) d s\right),
\end{aligned}
$$

where we have used the equality

$$
\int_{0}^{\infty} \theta^{\beta} \eta_{\alpha}(\theta) d \theta=\frac{\Gamma(1+\beta)}{\Gamma(1+\alpha \beta)} .
$$

Form (49) to (50), one can see that for each $z \in \mathbf{B}_{r}$,

$$
\left\|\left(\Theta_{\varepsilon} z\right)(t)-\left(\Theta_{\varepsilon}^{\lambda, \delta} z\right)(t)\right\| \longrightarrow 0 \quad \text { as } \lambda \longrightarrow 0^{+}, \delta \longrightarrow 0^{+} .
$$

Therefore, there are relatively compact sets arbitrary close to the set $\left\{\left(\Theta_{\varepsilon} z\right)(t): z \in \mathbf{B}_{r}\right\}$. Hence, the set $\left\{\left(\Theta_{\varepsilon} z\right)(t): z \in \mathbf{B}_{r}\right\}$ is also precompact in $\mathbf{B}_{r}$.

\section{Main Results}

Consider the following linear fractional differential system:

$$
\begin{gathered}
D_{t}^{\alpha} x(t)=A x(t)+B u(t), \quad t \in[0, T], \\
x(0)=\phi(0) .
\end{gathered}
$$

The approximate controllability for the linear fractional system (53) is a natural generalization of approximate controllability of linear first-order control system. It is convenient at this point to introduce the controllability operator associated with (53) as

$$
\begin{gathered}
L_{0}^{T}=\int_{0}^{T}(T-s)^{\alpha-1} \mathfrak{\Re}_{\alpha}(T-s) B u(s) d s, \\
\Gamma_{0}^{T}=L_{0}^{T}\left(L_{0}^{T}\right)^{*}=\int_{0}^{T}(T-s)^{2(\alpha-1)} \mathfrak{A}_{\alpha}(T-s) B B^{*} \mathfrak{\Re}_{\alpha}^{*}(T-s) d s,
\end{gathered}
$$

respectively, where $B^{*}$ denotes the adjoint of $B$ and $\mathfrak{P}_{\alpha}^{*}(t)$ is the adjoint of $\mathfrak{Y}_{\alpha}(t)$. It is straightforward that the operator $L_{0}^{T}$ is a linear-bounded operator for $1 / 2<\alpha \leq 1$.

Theorem 15 (see [11]). The following three conditions are equivalent:

(i) $\Gamma_{0}^{T}$ is positive, that is, $\left\langle z^{*}, \Gamma_{0}^{T} z^{*}\right\rangle>0$ for all nonzero $z^{*} \in X^{*}$.

(ii) For all $h \in X, J\left(z_{\varepsilon}(h)\right)$ converges to the zero as $\varepsilon \rightarrow 0^{+}$ in the weak topology, where $z_{\varepsilon}(h)=\varepsilon\left(\varepsilon I+\Gamma_{0}^{T} J\right)^{-1}(h)$ is a solution of the equation

$$
\varepsilon z_{\varepsilon}+\Gamma_{0}^{T} J\left(z_{\varepsilon}\right)=\alpha h .
$$

(iii) For all $h \in X, z_{\varepsilon}(h)=\varepsilon\left(\varepsilon I+\Gamma_{0}^{T} J\right)^{-1}(h)$ converges to the zero as $\varepsilon \rightarrow 0^{+}$in the strong topology.

Remark 16. It is known that Theorem 15(i) holds if and only if $\overline{\operatorname{Im} L_{0}^{T}}=X$. In other words, Theorem 15(i) holds if and only if the corresponding linear system is approximately controllable on $[0, T]$. 
Theorem 17 (see [11]). Let $p: X \rightarrow X$ be a nonlinear operator. Assume that $z_{\varepsilon}$ is a solution of the following equation:

$$
\begin{gathered}
\varepsilon z_{\varepsilon}+\Gamma_{0}^{T} J\left(z_{\varepsilon}\right)=\alpha p\left(z_{\varepsilon}\right), \\
\left\|p\left(z_{\varepsilon}\right)-p\right\| \longrightarrow 0 \text { as } \varepsilon \longrightarrow 0^{+}, p \in X .
\end{gathered}
$$

Then, there exists a subsequence of the sequence $\left\{z_{\varepsilon}\right\}$ strongly converging to zero as $\varepsilon \rightarrow 0^{+}$.

We are now in a position to state and prove the main result of the paper.

Theorem 18. Let $1 / 2<\alpha<1$. Suppose that conditions $\left(H_{1}\right)-$ $\left(H_{4}\right)$ and $\left(H_{a c}\right)$ are satisfied. Besides, assume additionally that

$\left(\mathrm{H}_{\mathrm{gc}}\right) g:[0, T] \times X \rightarrow X$ and $A^{\beta} g(T, \cdot)$ is continuous from the weak topology of $X$ to the strong topology of $X$.

$\left(\mathrm{H}_{\mathrm{ub}}\right)$ There exists $N \in L^{\infty}([0, T],[0,+\infty))$ such that

$\sup _{x \in B_{l}}\|f(t, x)\|+\sup _{y \in X}\left\|A^{\beta} g(t, y)\right\| \leq N(t), \quad$ for a.e. $t \in[0, T]$.

Then, the system (1) is approximately controllable on $[0, T]$.

Proof. Let $x^{\varepsilon}$ be a fixed point of $\Phi_{\varepsilon}$ in $B_{r(\varepsilon)}$. Then, $x^{\varepsilon}$ is a mild solution of (1) on $[0, T]$ under the control

$$
\begin{aligned}
u_{\varepsilon}\left(t, x^{\varepsilon}\right)= & (T-t)^{\alpha-1} B^{*} S^{*}(T-t) J\left(\left(\varepsilon I+\Gamma_{0}^{T} J\right)^{-1} p\left(x^{\varepsilon}\right)\right), \\
p\left(x^{\varepsilon}\right)= & h-\mathfrak{S}_{\alpha}(T)(\phi(0)+g(0, \phi(0)))+g\left(T, x^{\varepsilon}(T)\right) \\
& +\int_{0}^{T}(T-s)^{\alpha-1} A \mathfrak{A}_{\alpha}(T-s) g\left(s, x_{s}^{\varepsilon}\right) d s \\
& -\int_{0}^{T}(T-s)^{\alpha-1} \mathfrak{A}_{\alpha}(T-s) f\left(s, x_{s}^{\varepsilon}\right) d s
\end{aligned}
$$

and satisfies the following equality:

$$
\begin{aligned}
x^{\varepsilon}(T)= & \mathfrak{S}_{\alpha}(T)(\phi(0)+g(0, \phi(0)))-g\left(T, x^{\varepsilon}(T)\right) \\
& -\int_{0}^{T}(T-s)^{\alpha-1} A \mathfrak{A}_{\alpha}(T-s) g\left(s, x_{s}^{\varepsilon}\right) d s \\
& +\int_{0}^{T}(T-s)^{\alpha-1} \mathfrak{A}_{\alpha}(t-s)\left[B u_{\varepsilon}(s, x)+f\left(s, x_{s}^{\varepsilon}\right)\right] d s \\
= & \mathfrak{S}_{\alpha}(T)(\phi(0)+g(0, \phi(0)))-g\left(T, x^{\varepsilon}(T)\right) \\
& -\int_{0}^{T}(T-s)^{\alpha-1} A \mathfrak{A}_{\alpha}(T-s) h\left(s, x_{s}^{\varepsilon}\right) d s \\
& +\left(-\varepsilon I+\varepsilon I+\Gamma_{0}^{T} J\right)\left(\left(\varepsilon I+\Gamma_{0}^{T} J\right)^{-1} p\left(x^{\varepsilon}\right)\right) \\
& +\int_{0}^{T}(T-s)^{\alpha-1} \mathfrak{A}_{\alpha}(t-s) f\left(s, x_{s}^{\varepsilon}\right) d s \\
= & h-\varepsilon\left(\varepsilon I+\Gamma_{0}^{T} J\right)^{-1} p\left(x^{\varepsilon}\right) .
\end{aligned}
$$

In other words, $z_{\varepsilon}=h-x^{\varepsilon}(T)$ is a solution of the equation

$$
\left(\varepsilon I+\Gamma_{0}^{T} J\right)\left(z_{\varepsilon}\right)=\varepsilon p\left(x^{\varepsilon}\right) .
$$

It follows that

$$
\begin{gathered}
\varepsilon\left\langle J\left(z_{\varepsilon}\right), z_{\varepsilon}\right\rangle+\left\langle J\left(z_{\varepsilon}\right), \Gamma_{0}^{T} J\left(z_{\varepsilon}\right)\right\rangle=\varepsilon\left\langle J\left(z_{\varepsilon}\right), p\left(x^{\varepsilon}\right)\right\rangle, \\
\varepsilon\left\|z_{\varepsilon}\right\|^{2}+\left\langle J\left(z_{\varepsilon}\right), \Gamma_{0}^{T} J\left(z_{\varepsilon}\right)\right\rangle=\varepsilon\left\langle J\left(z_{\varepsilon}\right), p\left(x^{\varepsilon}\right)\right\rangle, \\
\varepsilon\left\|z_{\varepsilon}\right\|^{2} \leq \varepsilon\left\langle J\left(z_{\varepsilon}\right), p\left(x^{\varepsilon}\right)\right\rangle \leq \varepsilon\left\|z_{\varepsilon}\right\|\left\|p\left(x^{\varepsilon}\right)\right\|, \\
\left\|z_{\varepsilon}\right\|=\left\|J\left(z_{\varepsilon}\right)\right\| \leq\left\|p\left(x^{\varepsilon}\right)\right\| .
\end{gathered}
$$

On the other hand, by $\left(\mathrm{H}_{\mathrm{ub}}\right)$,

$$
\begin{aligned}
\left\|p\left(x^{\varepsilon}\right)\right\| \leq & \|h\|+M\|\phi(0)\|+N(T) \\
& +\frac{M_{1-\beta} \alpha \Gamma(1+\beta)}{\Gamma(1+\alpha \beta)} \int_{0}^{T}(T-s)^{\alpha \beta-1} N(s) d s \\
& +\frac{M}{\Gamma(\alpha)} \int_{0}^{T}(T-s)^{\alpha-1} N(s) d s .
\end{aligned}
$$

From (63) and (64), it follows that $x^{\varepsilon}(T) \rightarrow \tilde{x}$ weakly as $\varepsilon \rightarrow$ $0^{+}$and by the assumption $\left(\mathrm{H}_{\mathrm{gc}}\right) A^{\beta} g\left(T, x^{\varepsilon}(T)\right) \rightarrow A^{\beta} g(T, \tilde{x})$ strongly as $\varepsilon \rightarrow 0^{+}$. Moreover, because of assumption $\left(\mathrm{H}_{\mathrm{ub}}\right)$,

$$
\int_{0}^{T}\left\|f\left(s, x_{s}^{\varepsilon}\right)\right\|^{2} d s+\int_{0}^{T}\left\|A^{\beta} g\left(s, x_{s}^{\varepsilon}\right)\right\|^{2} d s \leq \int_{0}^{T} N(s) d s .
$$

Consequently, the sequences $\left\{f\left(\cdot, x^{\varepsilon}\right)\right\},\left\{A^{\beta} g\left(\cdot, x^{\varepsilon}\right)\right\}$ are bounded. Then, there is a subsequence still denoted by $\left\{f\left(\cdot, x^{\varepsilon}\right), A^{\beta} g\left(\cdot, x^{\varepsilon}\right)\right\}$ which weakly converges to, say, $(f(\cdot)$, $g(\cdot))$ in $L_{2}([0, T], X)$. Then,

$$
\begin{aligned}
& \left\|p\left(x^{\varepsilon}\right)-p\right\| \\
& =\left\|g\left(T, x^{\varepsilon}(T)\right)-g(T, \tilde{x})\right\| \\
& +\left\|\int_{0}^{T}(T-s)^{\alpha-1} A^{1-\beta} \mathfrak{U}_{\alpha}(T-s)\left[A^{\beta} g\left(s, x_{s}^{\varepsilon}\right)-g(s)\right] d s\right\| \\
& +\left\|\int_{0}^{T}(T-s)^{\alpha-1} \mathfrak{\Re}_{\alpha}(T-s)\left[f\left(s, x_{s}^{\varepsilon}\right)-f(s)\right] d s\right\| \\
& \leq\left\|A^{-\beta}\left(A^{\beta} g\left(T, x^{\varepsilon}(T)\right)-A^{\beta} g(T, \tilde{x})\right)\right\| \\
& +\sup _{0 \leq t \leq T}\left\|\int_{0}^{t}(t-s)^{\alpha-1} A^{1-\beta} \mathfrak{\Re}_{\alpha}(t-s)\left[A^{\beta} g\left(s, x_{s}^{\varepsilon}\right)-g(s)\right] d s\right\| \\
& \quad+\sup _{0 \leq t \leq T}\left\|\int_{0}^{t}(t-s)^{\alpha-1} \mathfrak{\Re}_{\alpha}(t-s)\left[f\left(s, x_{s}^{\varepsilon}\right)-f(s)\right] d s\right\| \longrightarrow 0,
\end{aligned}
$$


where

$$
\begin{aligned}
p & =h-\mathfrak{S}_{\alpha}(T)(\phi(0)+g(0, \phi(0)))+g(T, \tilde{x}) \\
& +\int_{0}^{T}(T-s)^{\alpha-1} A^{1-\beta} \mathfrak{A}_{\alpha}(T-s) g(s) d s \\
& -\int_{0}^{T}(T-s)^{\alpha-1} \mathfrak{A}_{\alpha}(T-s) f(s) d s
\end{aligned}
$$

as $\varepsilon \rightarrow 0^{+}$because of compactness of an operator $f(\cdot) \rightarrow$ $\int_{0}^{\cdot}(\cdot-s)^{\alpha-1} \mathfrak{A}_{\alpha}(\cdot-s) f(s) d s: L_{2}([0, T], X) \rightarrow C([0, T], X)$. Then, by Theorem 17

$$
\left\|x^{\varepsilon}(T)-h\right\|=\left\|z_{\varepsilon}\right\| \longrightarrow 0
$$

as $\varepsilon \rightarrow 0^{+}$. This gives the approximate controllability. The theorem is proved.

Remark 19. Theorem 18 assumes that the operator $A$ generates a compact semigroup. If the compactness condition holds on the bounded operator that maps the control function or the generated $C_{0}$-semigroup, then the controllability operator $L_{0}^{T}$ is also compact, and its inverse does not exist if the state space is infinite dimensional, and, consequently, the associated linear control system (53) is not exactly controllable. Therefore, the concept of complete controllability is too strong in infinite dimensional spaces, and the approximate controllability notion is more appropriate. Thus, Theorem 18 has no analogue for the concept of complete controllability.

\section{Applications}

In this section, we illustrate the obtained result. Let $X=$ $L_{2}[0, \pi]$, and let $A$ be defined as follows:

$$
A z=-z^{\prime \prime}
$$

with domain

$$
\begin{gathered}
D(A)=\left\{z \in X \mid z, \frac{d z}{d \xi}\right. \text { are absolutely continuous, } \\
\left.\frac{d^{2} z}{d \xi^{2}} \in X \text { and } z(0)=z(\pi)=0\right\} .
\end{gathered}
$$

Recall that $A$ is the infinitesimal generator of a strongly continuous semigroup $S(t), t>0$, on $X$ which is analytic compact and self-adjoint, and the eigenvalues are $-n^{2}, n \in$ $N$, with corresponding normalized eigenvectors $e_{n}(\xi):=$ $(2 / \pi)^{1 / 2} \sin (n \xi)$ and

$$
S(t) e_{n}=e^{-n^{2} t} e_{n}, \quad n=1,2, \ldots
$$

Moreover, the following hold.

(a) $\left\{e_{n}: n \in N\right\}$ is an orthonormal basis of $X$.

(b) If $z \in D(A)$, then $A(z)=\sum_{n=1}^{\infty} n^{2}\left\langle z, e_{n}\right\rangle e_{n}$.

(c) For $z \in \mathbb{H}, A^{-1 / 2} z=\sum_{n=1}^{\infty}(1 / n)\left\langle z, e_{n}\right\rangle e_{n}$. (d) The operator $A^{1 / 2}$ is given as $A^{1 / 2} z=\sum_{n=1}^{\infty} n\left\langle z, e_{n}\right\rangle e_{n}$ on the space $D\left[A^{1 / 2}\right]=\left\{z \in X: \sum_{n=1}^{\infty} n\left\langle z, e_{n}\right\rangle e_{n} \in X\right\}$.

For $1 / 2<\alpha<1$, consider the neutral system

$$
\begin{gathered}
\frac{\partial^{\alpha}}{\partial t^{\alpha}}\left[x(t, \xi)+\int_{0}^{\pi} b(\theta, \xi) x(t, \theta) \mathrm{d} \theta\right] \\
=\frac{\partial^{2}}{\partial \xi^{2}} x(t, \xi)+p(t, x(t, \xi))+B u(t, \xi), \\
x(t, 0)=x(t, \pi)=0, \quad t \geq 0, \\
x(0, \xi)=\varphi(\xi), \quad 0 \leq \xi \leq \pi,
\end{gathered}
$$

where $p:[0, T] \times R \rightarrow R$ is continuous functions. $B$ is a linear continuous mapping from $U=\left\{u=\sum_{n=2}^{\infty} u_{n} e_{n} \mid\|u\|_{U}^{2}:=\right.$ $\left.\sum_{n=2}^{\infty} u_{n}^{2}<\infty\right\}$ to $X$ as follows:

$$
B u=2 u_{2}+\sum_{n=2}^{\infty} u_{n} e_{n} .
$$

To write the initial-boundary value problem (72) in the abstract form, we assume the following.

(A1) The function $b$ is measurable and

$$
\int_{0}^{\pi} \int_{0}^{\pi} b^{2}(\theta, \xi) \mathrm{d} \theta \mathrm{d} \xi<\infty .
$$

(A2) The function $(\partial / \partial \xi) b(\theta, \xi)$ is measurable, $b(\theta, 0)=$ $b(\theta, \pi)=0$, and let

$$
L_{1}=\left[\int_{0}^{\pi} \int_{0}^{\pi}\left(\frac{\partial}{\partial \xi} b(\theta, \xi)\right)^{2} d \theta d \xi\right]^{1 / 2} .
$$

Define $f, g:[0, T] \times X \rightarrow X$ by

$$
\begin{gathered}
g(x)(\xi)=\int_{0}^{\pi} b(\theta, \xi) x(\theta) \mathrm{d} \theta, \\
f(t, x)(\xi)=p(t, x(\xi)) .
\end{gathered}
$$

From (A1), it is clear that $g$ is bounded linear operator on $X$. Furthermore, $g(x) \in D\left[A^{1 / 2}\right]$, and $\left\|A^{1 / 2} g\right\| \leq L_{1}$. In fact, from the definition of $g$ and (A2), it follows that

$$
\begin{aligned}
\left\langle g(x), e_{n}\right\rangle & =\int_{0}^{\pi}\left[\int_{0}^{\pi} b(\theta, \xi) x(\theta) \mathrm{d} \theta\right] e_{n}(\xi) \mathrm{d} \xi \\
& =\frac{1}{n}\left(\frac{2}{\pi}\right)^{1 / 2}\left\langle\int_{0}^{\pi} \frac{\partial}{\partial \xi} b(\theta, \xi) x(\theta) \mathrm{d} \theta, \cos (n \xi)\right\rangle \\
& =\frac{1}{n}\left(\frac{2}{\pi}\right)^{1 / 2}\left\langle g_{1}(x), \cos (n \xi)\right\rangle,
\end{aligned}
$$

where $g_{1}(x)=\int_{0}^{\pi}(\partial / \partial \xi) b(\theta, \xi) x(\theta) \mathrm{d} \theta$. From (A2), we know that $g_{1}: X \rightarrow X$ is a bounded linear operator with $\left\|g_{1}\right\| \leq L_{1}$. Hence, $\left\|A^{1 / 2} g(x)\right\|=\left\|g_{1}(x)\right\|$, which implies the 
assertion. Moreover, assume that $f$ and $g$ satisfy conditions of Theorem 18. Thus, the problem (72) can be written in the abstract form

$$
\begin{gathered}
\frac{d^{\alpha}}{d t^{\alpha}}(x(t)+g(t, x(t)))=A x(t)+f(t, x(t))+B u(t), \\
x(0)=x_{0}, \quad t \in[0, T] .
\end{gathered}
$$

Now, consider the associated linear system

$$
\begin{gathered}
\frac{d^{\alpha}}{d t^{\alpha}} x(t)=A x(t)+B u(t), \\
x(0)=x_{0}, \quad t \in[0, T] .
\end{gathered}
$$

Show that it is approximately controllable on $[0, T]$ for $1 / 2<$ $\alpha<1$. It is easy to see that if $z=\sum_{n=1}^{\infty}\left\langle z, e_{n}\right\rangle e_{n}$, then

$$
B^{*} v=\left(2 v_{1}+v_{2}\right) e_{2}+\sum_{n=3}^{\infty} v_{n} e_{n}
$$

$$
\begin{aligned}
& B^{*} \mathfrak{\mathcal { A }}_{\alpha}^{*}(T-s) z \\
& =B^{*} \alpha \int_{0}^{\infty} \theta \Psi_{\alpha}(\theta) S^{*}\left((T-s)^{\alpha} \theta\right) z d \theta \\
& =\alpha \int_{0}^{\infty} \theta \Psi_{\alpha}(\theta)\left(\left(2\left\langle z, e_{1}\right\rangle e^{-(T-s)^{\alpha} \theta} e_{1}+\left\langle z, e_{2}\right\rangle e^{-4(T-s)^{\alpha} \theta}\right) e_{2}\right. \\
& \left.+\sum_{n=3}^{\infty} e^{-n^{2}(T-s)^{\alpha} \theta}\left\langle z, e_{n}\right\rangle e_{n}\right) d \theta \\
& =\left(2\left\langle z, e_{1}\right\rangle \alpha \int_{0}^{\infty} \theta \Psi_{\alpha}(\theta) e^{-(T-s)^{\alpha} \theta} d \theta\right. \\
& \left.+\left\langle z, e_{2}\right\rangle \alpha \int_{0}^{\infty} \theta \Psi_{\alpha}(\theta) e^{-4(T-s)^{\alpha} \theta} d \theta\right) e_{2} \\
& +\alpha \sum_{n=3}^{\infty} \int_{0}^{\infty} \theta \Psi_{\alpha}(\theta) e^{-n^{2}(T-s)^{\alpha} \theta} d \theta\left\langle z, e_{n}\right\rangle e_{n} \\
& \left\|(T-s)^{\alpha-1} B^{*} \mathfrak{A}_{\alpha}^{*}(T-s) z\right\|^{2} \\
& =(T-s)^{2(\alpha-1)}\left(2 \alpha \int_{0}^{\infty} \theta \Psi_{\alpha}(\theta) e^{-(T-s)^{\alpha} \theta} d \theta\left\langle z, e_{1}\right\rangle\right. \\
& \left.+\alpha \int_{0}^{\infty} \theta \Psi_{\alpha}(\theta) e^{-4(T-s)^{\alpha} \theta} d \theta\left\langle z, e_{2}\right\rangle\right)^{2} \\
& +(T-s)^{2(\alpha-1)} \sum_{n=3}^{\infty}\left(\alpha \sum_{n=3}^{\infty} \int_{0}^{\infty} \theta \Psi_{\alpha}(\theta) e^{-n^{2}(T-s)^{\alpha} \theta} d \theta\right)^{2} \\
& \times\left\langle z, e_{n}\right\rangle^{2}=0 \text {. }
\end{aligned}
$$

It follows that $\left\langle z, e_{1}\right\rangle=\left\langle z, e_{2}\right\rangle=\cdots=\left\langle z, e_{n}\right\rangle=\cdots=0$, and consequently, $z=0$, which means that (79) is approximately controllable on $[0, T]$. Therefore, from Theorem 18 , the system (72) is approximately controllable $[0, T]$.

\section{Conclusion}

In this paper, abstract results concerning the approximate controllability of fractional semilinear evolution systems with infinite delay in a separable reflexive Banach space are obtained. Approximate controllability result for semilinear systems is obtained by means of the Krasnoselskii's fixedpoint theorem under the compactness assumption. It is also proven that the controllability of the semilinear system is implied by the approximate controllability of the associated linear system under some natural conditions. Upon making some appropriate assumptions, by employing the ideas and techniques as in this paper, one can establish the approximate controllability results for a wide class of fractional deterministic and stochastic evolution equations.

\section{Acknowledgment}

N. I. Mahmudov is thankful to the reviewers for making valuable suggestions leading to the better presentation of this paper.

\section{References}

[1] S. G. Samko, A. A. Kilbas, and O. I. Marichev, Fractional Integrals and Derivatives. Theory and Applications, Gordon and Breach Science, Yverdon, Switzerland, 1993.

[2] R. F. Curtain and H. J. Zwart, An Introduction to InfiniteDimensional Linear Systems Theory, Springer, Berlin, Germany, 1995.

[3] A. Debbouche and D. Baleanu, "Controllability of fractional evolution nonlocal impulsive quasilinear delay integro-differential systems," Computers \& Mathematics with Applications, vol. 62, no. 3, pp. 1442-1450, 2011.

[4] K. Balachandran and J. P. Dauer, "Controllability of nonlinear systems in Banach spaces: a survey," Journal of Optimization Theory and Applications, vol. 115, no. 1, pp. 7-28, 2002.

[5] A. E. Bashirov and N. I. Mahmudov, "On concepts of controllability for deterministic and stochastic systems," SIAM Journal on Control and Optimization, vol. 37, no. 6, pp. 1808-1821, 1999.

[6] M. Benchohra, L. Górniewicz, and S. K. Ntouyas, Controllability of Some Nonlinear Systems in Banach Spaces: The Fixed Point Theory Approach, Pawel Wlodkowicz University College, Plock, Poland, 2003.

[7] N. Carmichael and M. D. Quinn, "Fixed point methods in nonlinear control," in Distributed Parameter Systems (Vorau, 1984), vol. 75 of Lecture Notes in Control and Information Sciences, pp. 24-51, Springer, Berlin, Germany, 1985.

[8] D. N. Chalishajar, "Controllability of second order impulsive neutral functional differential inclusions with infinite delay," Journal of Optimization Theory and Applications, vol. 154, no. 2, pp. 672-684, 2012.

[9] D. N. Chalishajar and F. S. Acharya, "Controllability of second order semi-linear neutral impulsive differential inclusions on unbounded domain with infinite delay in Banach spaces," Bulletin of the Korean Mathematical Society, vol. 48, no. 4, pp. 813-838, 2011.

[10] X. J. Li and J. M. Yong, Optimal Control Theory for InfiniteDimensional Systems, Systems \& Control: Foundations \& Applications, Birkhäuser Boston Publishers, Boston, Mass, USA, 1995. 
[11] N. I. Mahmudov, "Approximate controllability of semilinear deterministic and stochastic evolution equations in abstract spaces," SIAM Journal on Control and Optimization, vol. 42, no. 5, pp. 1604-1622, 2003.

[12] J. P. Dauer and N. I. Mahmudov, "Approximate controllability of semilinear functional equations in Hilbert spaces," Journal of Mathematical Analysis and Applications, vol. 273, no. 2, pp. 310327, 2002.

[13] J. Klamka, "Local controllability of fractional discrete-time semilinear systems," Acta Mechanica et Automatica, vol. 15, pp. 55-58, 2011.

[14] S. Kumar and N. Sukavanam, "Approximate controllability of fractional order semilinear systems with bounded delay," Journal of Differential Equations, vol. 252, no. 11, pp. 6163-6174, 2012.

[15] N. I. Mahmudov, "Approximate controllability of evolution systems with nonlocal conditions," Nonlinear Analysis. Theory, Methods \& Applications, vol. 68, no. 3, pp. 536-546, 2008.

[16] V. Obukhovskiǔ and P. Rubbioni, "On a controllability problem for systems governed by semilinear functional differential inclusions in Banach spaces," Topological Methods in Nonlinear Analysis, vol. 15, no. 1, pp. 141-151, 2000.

[17] V. Obukhovski and P. Zecca, "Controllability for systems governed by semilinear differential inclusions in a Banach space with a noncompact semigroup," Nonlinear Analysis. Theory, Methods \& Applications, vol. 70, no. 9, pp. 3424-3436, 2009.

[18] R. Sakthivel, J. J. Nieto, and N. I. Mahmudov, "Approximate controllability of nonlinear deterministic and stochastic systems with unbounded delay," Taiwanese Journal of Mathematics, vol. 14, no. 5, pp. 1777-1797, 2010.

[19] R. Sakthivel, N. I. Mahmudov, and Juan. J. Nieto, "Controllability for a class of fractional-order neutral evolution control systems," Applied Mathematics and Computation, vol. 218, no. 20, pp. 10334-10340, 2012.

[20] R. Sakthivel and Y. Ren, "Approximate controllability of fractional differential equations with state-dependent delay," Results in Mathematics, 2012.

[21] R. Sakthivel, Y. Ren, and N. I. Mahmudov, "On the approximate controllability of semilinear fractional differential systems," Computers \& Mathematics with Applications, vol. 62, no. 3, pp. 1451-1459, 2011.

[22] Y. Ren, L. Hu, and R. Sakthivel, "Controllability of impulsive neutral stochastic functional differential inclusions with infinite delay," Journal of Computational and Applied Mathematics, vol. 235, no. 8, pp. 2603-2614, 2011.

[23] R. Sakthivel, S. Suganya, and S. M. Anthoni, "Approximate controllability of fractional stochastic evolution equations," Computers \& Mathematics with Applications, vol. 63, no. 3, pp. 660-668, 2012.

[24] R. Sakthivel and Y. Ren, "Complete controllability of stochastic evolution equations with jumps," Reports on Mathematical Physics, vol. 68, no. 2, pp. 163-174, 2011.

[25] R. Sakthivel, N. I. Mahmudov, and J. H. Kim, "On controllability of second order nonlinear impulsive differential systems," Nonlinear Analysis. Theory, Methods \& Applications, vol. 71, no. 1-2, pp. 45-52, 2009.

[26] R. Sakthivel, "Controllability of nonlinear impulsive Ito type stochastic systems," International Journal of Applied Mathematics and Computer Science, vol. 19, no. 4, pp. 589-595, 2009.

[27] N. Sukavanam and S. Kumar, "Approximate controllability of fractional order semilinear delay systems," Journal of Optimization Theory and Applications, vol. 151, no. 2, pp. 373-384, 2011.
[28] Z. Yan, "Approximate controllability of partial neutral functional differential systems of fractional order with statedependent delay," International Journal of Control, vol. 85, no. 8, pp. 1051-1062, 2012.

[29] J. Wang and Y. Zhou, "Complete controllability of fractional evolution systems," Communications in Nonlinear Science and Numerical Simulation, vol. 17, no. 11, pp. 4346-4355, 2012.

[30] J. Wang, Z. Fan, and Y. Zhou, "Nonlocal controllability of semilinear dynamic systems with fractional derivative in Banach spaces," Journal of Optimization Theory and Applications, vol. 154, no. 1, pp. 292-302, 2012.

[31] A. Pazy, Semigroups of Linear Operators and Applications to Partial Differential Equations, vol. 44 of Applied Mathematical Sciences, Springer, New York, NY, USA, 1983.

[32] Y. Zhou and F. Jiao, "Existence of mild solutions for fractional neutral evolution equations," Computers \& Mathematics with Applications, vol. 59, no. 3, pp. 1063-1077, 2010.

[33] Y. Zhou and F. Jiao, "Nonlocal Cauchy problem for fractional evolution equations," Nonlinear Analysis. Real World Applications, vol. 11, no. 5, pp. 4465-4475, 2010.

[34] J. Wang and Y. Zhou, "A class of fractional evolution equations and optimal controls," Nonlinear Analysis. Real World Applications, vol. 12, no. 1, pp. 262-272, 2011. 


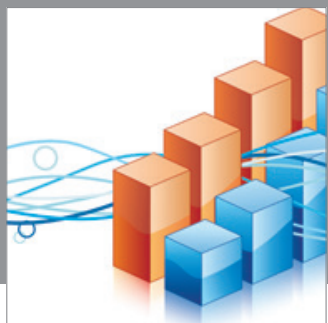

Advances in

Operations Research

mansans

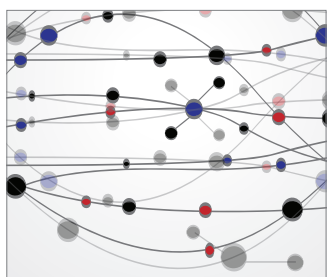

The Scientific World Journal
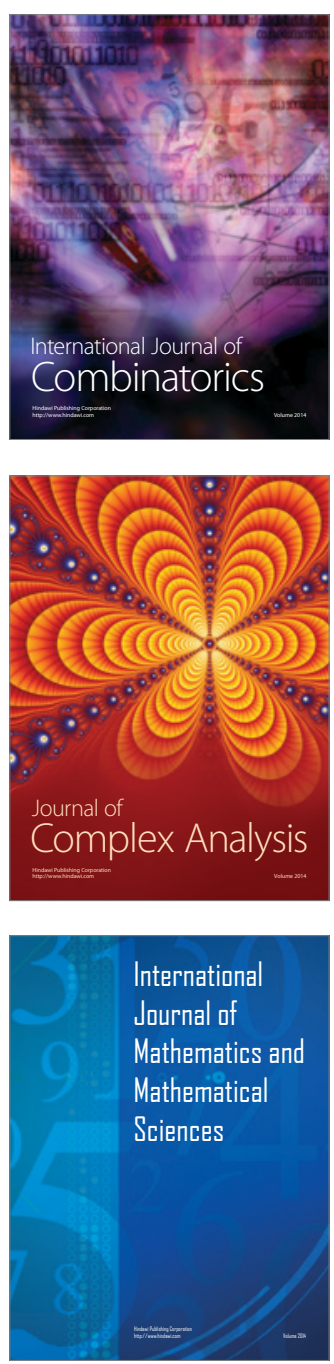
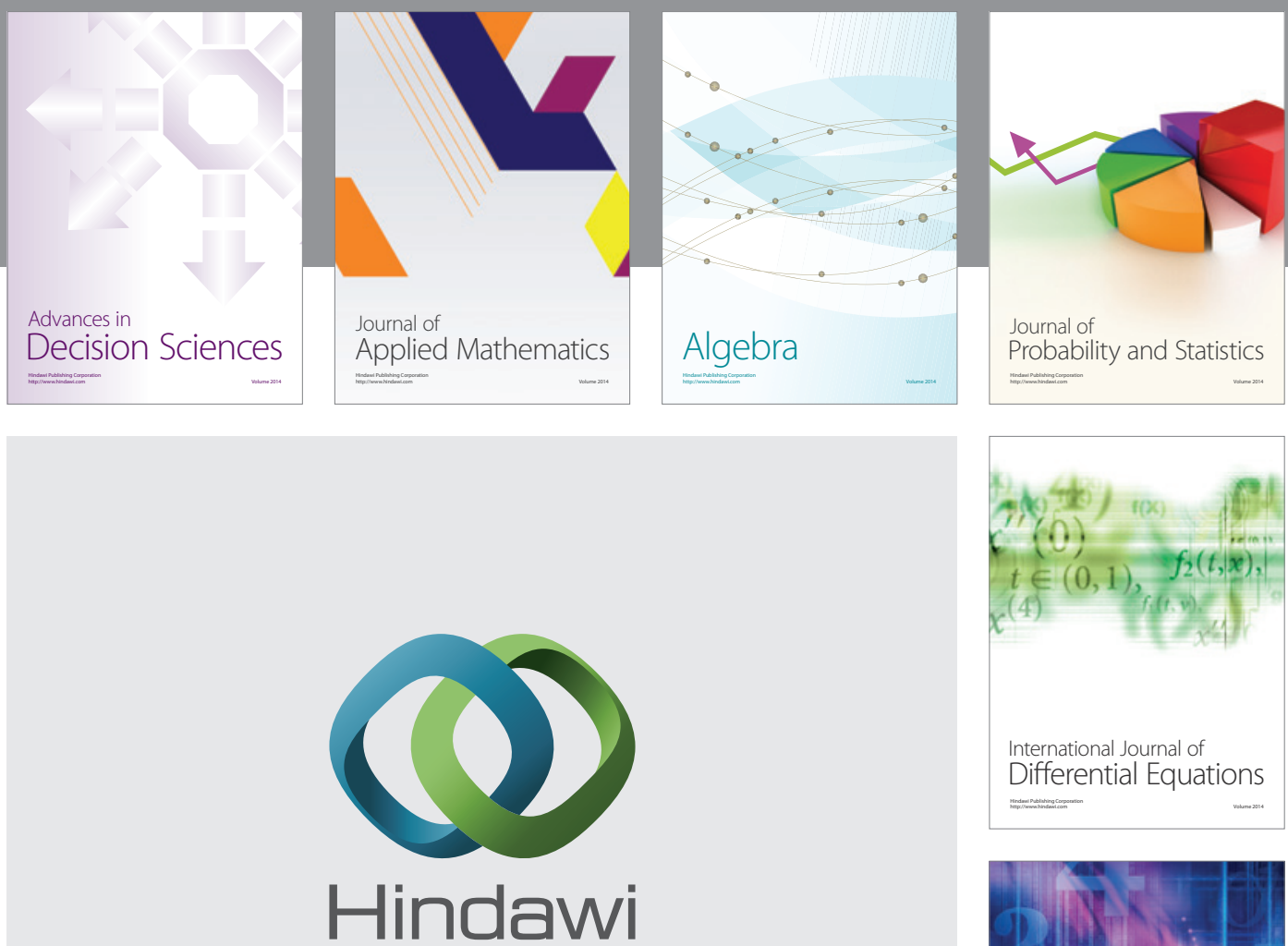

Submit your manuscripts at http://www.hindawi.com
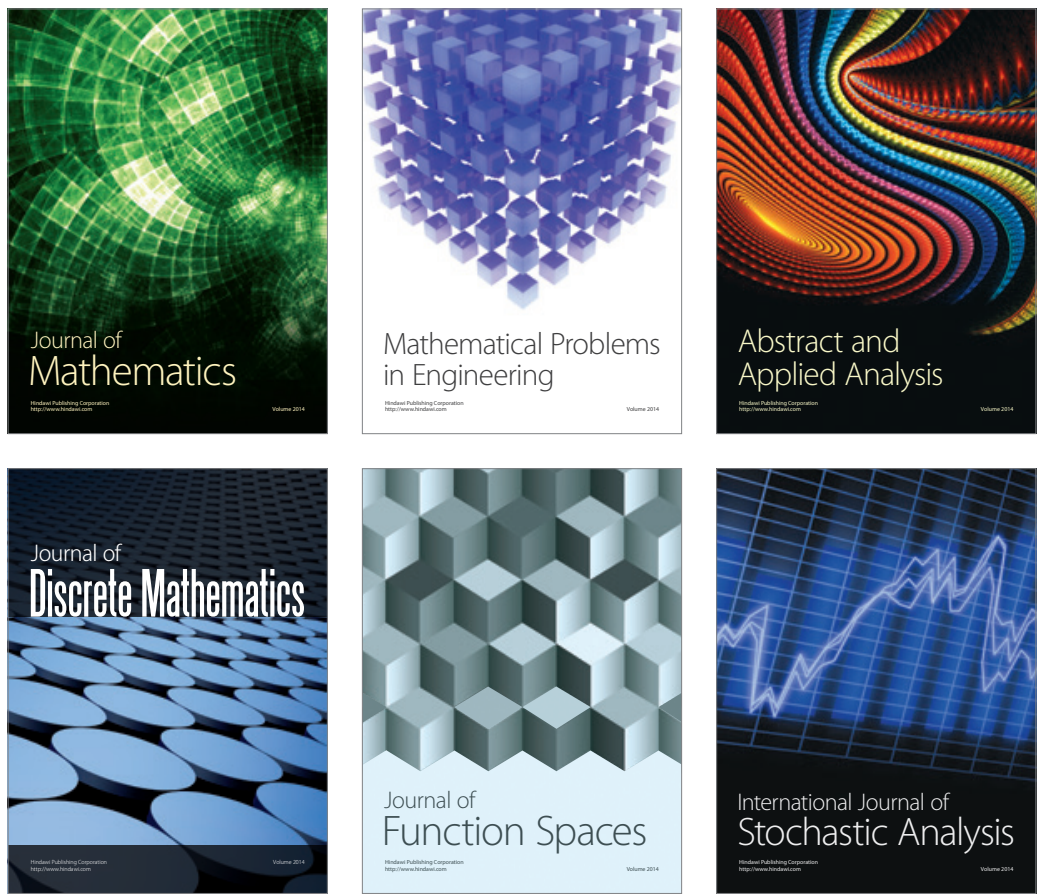

Journal of

Function Spaces

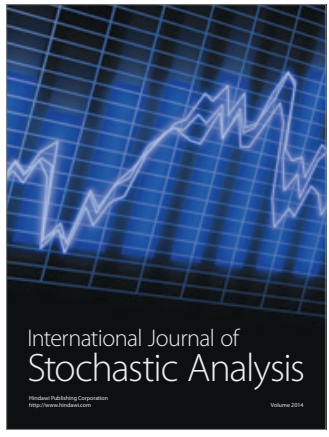

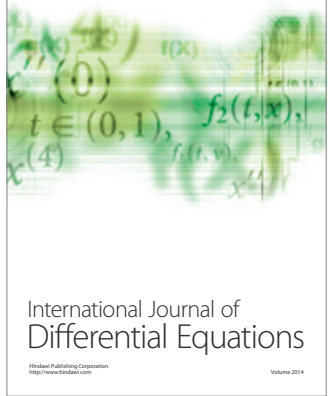
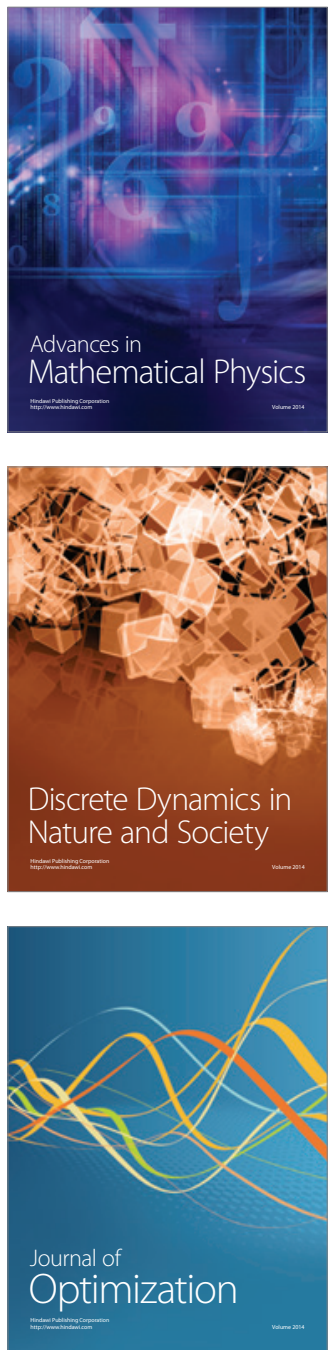